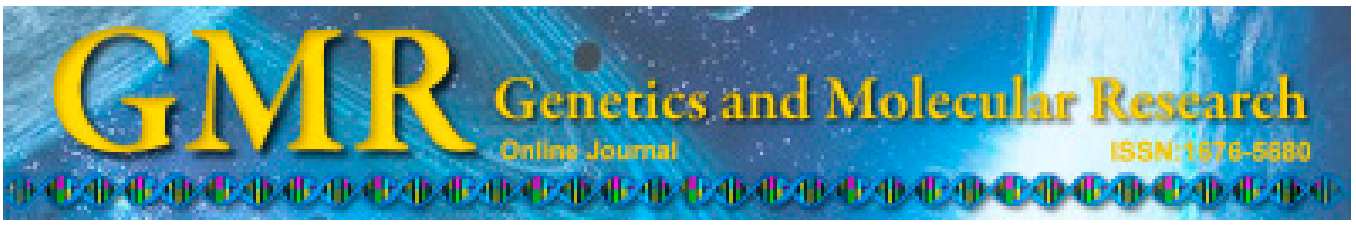

\title{
Comparison between meat quality of Hanzhong White pigs and carcass weight
}

\author{
G.F. Wu ${ }^{1,2}$, X.E. Shi ${ }^{1}$, Y.F. Li ${ }^{1}$, H.Z. Lu ${ }^{1}$, C.C. Song ${ }^{1}$, L. Jia ${ }^{1}$ and S.D. Sun ${ }^{1}$ \\ ${ }^{1}$ College of Animal Science and Technology, Northwest A\&F University, \\ Yangling, China \\ ${ }^{2}$ Qinghai Academy of Animal Science and Veterinary Medicine, \\ Qinghai University, Xining, China \\ Corresponding author: S.D. Sun \\ E-mail:ssdsm@tom.com
}

Genet. Mol. Res. 14 (2): 6059-6064 (2015)

Received February 27, 2014

Accepted September 12, 2014

Published June 8, 2015

DOI http://dx.doi.org/10.4238/2015.June.8.3

\begin{abstract}
A total of 48 carcasses of crossbred Hanzhong White pigs were divided into 3 groups (I, 90-99 kg; II, 100-109 kg; III, 110$119 \mathrm{~kg}$ ) to investigate the influence of carcass weight on meat quality. The intramuscular fat content of the 3 groups increased from 1.90 to $4.90 \%$; for meat color, Warner-Bratzler shear force, drip loss, and oxidation-type muscle fiber percentage, and muscle fiber diameter of the longissimus lumborum, the indices in group II and group III were better than those in group I $(\mathrm{P}<0.05)$. The saturated fatty acid and polyunsaturated fatty acid percentages of the longissimus lumborum muscle (2.80 and $37.30 \%$, respectively) in group II were significantly lower than those in the other 2 groups, while the monounsaturated fatty acid percentage was the highest $(59.10 \%)$. In conclusion, our results suggest that a carcass weight of $100-109 \mathrm{~kg}$ is sufficient to produce acceptable meat quality of Hanzhong White pigs.
\end{abstract}

Key words: Carcass weight; Fatty acid composition; Meat quality;

Oxidative type muscle fiber; Hanzhong White pig 


\section{INTRODUCTION}

Hanzhong White pig (HZWP) is a developed pig breed in China's Shaanxi Province; HZWP is a crossbreed of Su White x Berkshire x Hanjiang Black pig and has a slower rate of growth than commercial pig breeds (Zhao and Liu, 2007; Wang et al., 2008). The meat of HZWP shows higher redness and intramuscular fat (IMF) content compared with the commercial pig breeds (Li et al., 1991). However, few scientific studies have examined the meat quality of HZWP, particularly with respect to the relationships between muscle fiber characteristics, IMF content, and fatty acid composition.

An important component of meat quality assessment is identifying the muscle microstructure. It is widely accepted that one of the main factors determining physicochemical traits is fiber-type composition. Postmortem changes affect fiber-type composition in the conversion of muscle to meat, and thus affect meat quality (Ryu and Kim, 2006). In addition to its cognitive significance, determining the associations between muscle microstructure with physicochemical properties of muscle will increase the understanding of meatiness and regulation of meat characteristics. In pigs, increasing the cross-sectional area of muscle fiber is thought to be detrimental to meat quality, particularly its water-holding capacity and tenderness (Rehfeldt et al., 2000). However, IMF content and fatty acid composition are also important factors affecting the nutritional quality of muscle, and have been widely studied in meat science because of their important role in human health (Raes et al., 2004; Teye et al., 2006). Different breeds have variable suitable slaughter weights. The purpose of this study was to investigate the relationship between carcass weight and meat quality in HZWP.

\section{MATERIAL AND METHODS}

All experimental procedures were performed according to the Guide for Animal Care and Use of Laboratory Animals in the Institutional Animal Care and Use Committee of Northwest A\&F University. The experimental protocol was approved by the Department Animal Ethics Committee of Northwest A\&F University.

\section{Animals and management}

A total of 48 HZBPs, ranging from $90-109 \mathrm{~kg}$ in carcass weight, were selected and divided into 3 carcass weight groups (16 carcasses each): I, 90-99 kg (6 months old); II, 100-109 $\mathrm{kg}$ (7 months old); III, 110-119 kg (8 months old). All pigs were fed ad libitum with a commercial diet (Table S1) suited to their nutritive needs. The pigs were kept off feed and given free access to water for $16 \mathrm{~h}$, and then electrically stunned, exsanguinated, scalded, and rinsed.

\section{Carcass and meat quality measurements}

At 45 min after slaughter, $\mathrm{pH}$ was measured using a portable $\mathrm{pH}$ meter, and meat color was estimated based on pork quality standards (American NPPC shade guide, $1994 \mathrm{edn}$.); Warner-Bratzler shear force (WBSF) was measured using a C-LM3 Digital Display tenderness instrument equipped with a shearing device (XIELI Sci. Co., Ltd., Harbin, China) (Zhang et al., 2010). After 24-h ageing of the carcasses, the longissimus lumborum muscles were isolated to evaluate drip loss using the method described by Honikel (1998), with modifications: 
muscle samples standardized for surface area $\left(5 \times 3 \times 2.5 \mathrm{~cm}^{3}\right)$ were suspended in an inflated plastic bag for $24 \mathrm{~h}$ at $4^{\circ} \mathrm{C}$.

\section{Muscle fiber type and size}

The composition and size of oxidative muscle fiber type was determined by immunofluorescence (Kim et al., 2013), Troponin I-SS antibody (Santa Cruz Biotechnologies, Santa Cruz, CA, USA) was used to label oxidative muscle fiber, and nuclei were stained with 4',6-diamidino-2-phenylindole. Images were visualized and captured using a Nikon TE 2000 microscope (Nikon, Tokyo, Japan), and approximately 600 fibers per sample were counted.

\section{Fatty acid composition analyses}

The muscle sample was isolated from the fourth to fifth intercostal space. IMF content was determined using the Weibull-Stoldt method AOAC (1997). Fatty acids from the longissimus lumborum muscle were detected as their methyl esters according to Park and Goins (1994). Separation of fatty acid methyl esters was performed by gas chromatography using a Tracegc Ultra system (Thermo Scientific, Waltham, MA, USA), while fatty acid composition was calculated using response factors derived from chromatographic standards (GLC-91, NUCHEK, Elysian, MN, USA) of known composition. The results are reported as a percentage of the total fatty acid content.

\section{Statistical analyses}

Statistical analysis was performed using SAS version 8.0 (SAS Inst. Inc., Cary, NC, USA). Differences were considered to be significant when $\mathrm{P} \leq 0.05$.

\section{RESULTS AND DISCUSSION}

\section{Meat quality traits}

IMF significantly increased with carcass weight $(\mathrm{P}<0.05$; Table 1$)$. Ultimate $\mathrm{pH}$ showed no difference among the 3 groups, while the meat color value was significantly different between group I and group II $(\mathrm{P}<0.05$; Table 1$)$, which was in accordance with the results of previous studies (Correa et al., 2006; Galián et al., 2009).

Table 1. Meat quality traits in the 3 groups of HWZPs.

\begin{tabular}{lcrr}
\hline & & Groups & \\
\cline { 2 - 4 } & I (6-month-old pigs) & II (7-month-old pigs) & III (8-month-old pigs) \\
\hline Carcass weight (kg) & $96.70 \pm 1.53^{\mathrm{a}}$ & $105.40 \pm 2.71^{\mathrm{b}}$ & $114.20 \pm 2.06^{\mathrm{c}}$ \\
Ultimate pH & $6.43 \pm 0.35^{\mathrm{a}}$ & $6.42 \pm 0.27^{\mathrm{a}}$ & $6.50 \pm 0.71^{\mathrm{a}}$ \\
Meat color & $2.50 \pm 0.14^{\mathrm{a}}$ & $3.50 \pm 0.17^{\mathrm{b}}$ & $3.50 \pm 0.11^{\mathrm{b}}$ \\
IMF content (\%) & $1.90 \pm 0.21^{\mathrm{a}}$ & $3.70 \pm 0.12^{\mathrm{b}}$ & $4.90 \pm 0.32^{\mathrm{c}}$ \\
WBSF (N) & $36.50 \pm 1.81^{\mathrm{a}}$ & $26.00 \pm 2.74^{\mathrm{b}}$ & $25.21 \pm 1.30^{\mathrm{b}}$ \\
Drip loss (\%) & $2.70 \pm 0.35^{\mathrm{a}}$ & $1.50 \pm 0.19^{\mathrm{b}}$ & $1.30 \pm 0.23^{\mathrm{b}}$ \\
\hline
\end{tabular}

Means \pm SE with different letters $(a, b, c)$ are significantly different $(\mathrm{P}<0.05)$. WBSF $=$ Warner-Bratzler shear force; IMF = intramuscular fat. 
Oxidative muscle fiber composition $(9.2,16.8$, and $18.1 \%$ for groups I, II and III, respectively) increased with increasing carcass weight; values were significantly different between group I and group II $(\mathrm{P}<0.05)$, but not between group II and group III (Figure 1). The muscle fiber diameters in the 3 groups were $39.8,31.5$, and $29.7 \mu \mathrm{m}$ for groups I, II and III, respectively, indicating an opposite trend relative to oxidation type and muscle fiber composition. Ryu and Kim (2005) reported that fiber number and area percentage were not affected by carcass weight and loin-eye area. Other studies showed that pigs with greater weights have the highest proportion of fiber type IIB, and thus the proportion of type I and IIA fibers was decreased (Bee, 2004). However, we obtained opposite results, finding that heavier carcass weights were associated with higher percentages of oxidative muscle fiber. This may be because we used immunofluorescence staining with antibody selection to label the myosin heavy chain I gene. Another reason may be related to characteristics specific to the HZWP breed. Future studies should be conducted to assess the changes in muscle fiber type at different ages.

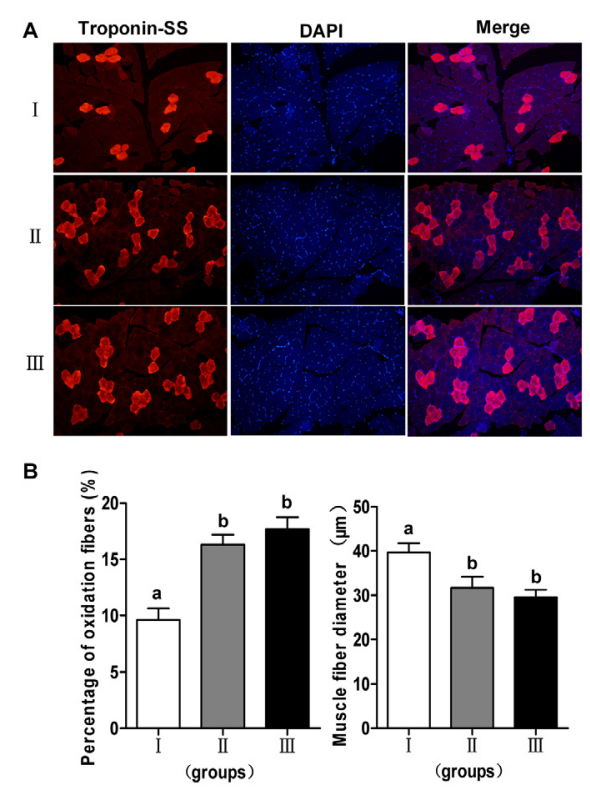

Figure 1. Determination of oxidative muscle fiber composition of the longissimus lumborum (LL) muscle in the 3 groups. A. Immunofluorescence staining with Troponin-SS for oxidation fibers. Troponin-SS staining (red); DAPI staining (blue) (100X). B. Quantification of oxidation fibers and muscle fiber diameter of LL muscle from the 3 carcass groups. Data are reported as means \pm standard error of the mean; different letters $(a, b, c)$ indicate significantly different results $(\mathrm{P}<0.05)$.

\section{Fatty acid composition of IMF in the longissimus lumborum muscle}

A summary of the fatty acid profile and selected ratios are shown in Table 2. Compared with the other 2 groups, group II contained large amounts of palmitoleic acid and oleic acid, but less palmitic, stearic, and linoleic acid. The fatty acid composition of the longissimus lumborum muscle showed that the saturated fatty acid and polyunsaturated fatty acid percentages (37.30 and $2.80 \%$, respectively) in group II were significantly lower than those in the other 2 groups, while the monounsaturated fatty acid percentage was the highest $(59.10 \%)$, 
mainly including oleic acid. Group II showed the lowest polyunsaturated fatty acid/saturated fatty acid (P:S) ratio $(<0.05)$. The content of the 2 major monounsaturated fatty acids (oleic and palmitoleic acids) increased with increasing fat content (Table 2), which is consistent with the results of other studies (Furman et al., 2010). Different weights showed different fatty acid ratios. We found that group I had the lowest IMF percentage $(1.9 \%)$ and highest P:S ratio (11.67) and linoleic acid content (C18:2n-6) (4.70\%) (Table 2). Kouba et al. (2003) and Furman et al. (2010) found similar trends for $\mathrm{C} 16: 1 \mathrm{n}-7$ and $\mathrm{C} 18: 1 \mathrm{n}-9$ in the longissimus muscle with increasing age and fattiness of pigs. A higher P:S ratio was observed for lower IMF content in commercial pigs. De Smet et al. (2004) found higher contents of saturated fatty acid and monounsaturated fatty acid than polyunsaturated fatty acid with increasing fattiness, leading to a decrease in the P:S ratio. These studies support our experimental results.

Table 2. Fatty acid contents of the longissimus lumborum muscle in the 3 groups.

\begin{tabular}{|c|c|c|c|c|}
\hline & Groups & I & II & III \\
\hline \multicolumn{2}{|l|}{ SFA $(\%)$} & $40.20 \pm 1.37^{\mathrm{a}}$ & $37.3 \pm 0.74^{\mathrm{b}}$ & $38.4 \pm 0.62^{\mathrm{b}}$ \\
\hline C14:0 & Myristic acid & $1.20 \pm 0.19^{\mathrm{a}}$ & $1.30 \pm 0.18^{\mathrm{ab}}$ & $1.40 \pm 0.12^{\mathrm{b}}$ \\
\hline C16:0 & Palmitic acid & $26.00 \pm 2.13^{\mathrm{a}}$ & $25.00 \pm 2.38^{\mathrm{a}}$ & $26.00 \pm 1.85^{\mathrm{a}}$ \\
\hline C18:0 & Stearic acid & $13.00 \pm 0.73^{\mathrm{a}}$ & $11.00 \pm 0.82^{\mathrm{a}}$ & $11.00 \pm 0.47^{\mathrm{a}}$ \\
\hline \multicolumn{2}{|l|}{ MUFA (\%) } & $53.90 \pm 3.49^{\mathrm{a}}$ & $59.10 \pm 2.76^{\mathrm{b}}$ & $55.00 \pm 3.17^{\mathrm{c}}$ \\
\hline C16:1n-7 & Palmitoleic acid & $3.90 \pm 0.52^{\mathrm{a}}$ & $5.10 \pm 0.37^{b}$ & $5.00 \pm 0.29^{b}$ \\
\hline C18:1n-9 & Oleic acid & $50.00 \pm 1.75^{\mathrm{a}}$ & $54.00 \pm 1.59^{\mathrm{b}}$ & $50.00 \pm 1.91^{\mathrm{a}}$ \\
\hline \multicolumn{2}{|l|}{ PUFA (\%) } & $4.70 \pm 0.15^{\mathrm{a}}$ & $2.80 \pm 0.11^{\mathrm{b}}$ & $4.10 \pm 0.37^{\mathrm{a}}$ \\
\hline $\mathrm{C} 18: 2 \mathrm{n}-6$ & Linoleic acid & $4.70 \pm 0.15^{\mathrm{a}}$ & $2.80 \pm 0.11^{\mathrm{b}}$ & $4.10 \pm 0.37^{\mathrm{a}}$ \\
\hline \multicolumn{2}{|c|}{ PUFA/SFA (P:S) ratio } & $11.67 \pm 0.21^{\mathrm{a}}$ & $7.50 \pm 0.13^{\mathrm{b}}$ & $10.60 \pm 0.17^{\mathrm{a}}$ \\
\hline
\end{tabular}

Means \pm SE with different letters $(a, b, c)$ are significantly different $(\mathrm{P}<0.05)$. SFA = saturated fatty acids; MUFA $=$ monounsaturated fatty acids; PUFA $=$ polyunsaturated fatty acids .

\section{CONCLUSIONS}

In conclusion, the carcass weight of HZWP is related to muscle fiber characteristics and has an influence on meat quality. In HZWP, the longissimus lumborum muscle of heavier carcass weight pigs showed a higher percentage of oxidative-type muscle fibers. The loineye area and IMF were also increased with increasing carcass weight, and IMF percentage affected fatty acid composition. Therefore, excessively high carcass weight influences meat quality because of muscle fiber characteristics, particularly oxidative muscle fiber. The suitable slaughter weight of HZWP ranged from 100-109 kg.

\section{ACKNOWLEDGMENTS}

Research supported by the National Natural Science Foundation of China (Grant \#31272410), the Scientific and Technological Innovation Project for Youths of Northwest A\&F University (Grant \#ZD2012007), and the National Swine Industry Technology System (Grant \#CARS-36-04B).

\section{Supplementary material}

\section{REFERENCES}

AOAC, (1997). Official Methods of Analysis, 16th edn. Association of Official Analytical Chemists, AOAC International, Gaithersburg 
Bee G (2004). Effect of early gestation feeding, birth weight, and gender of progeny on muscle fiber characteristics of pigs at slaughter. J. Anim. Sci. 82: 826-836.

Correa JA, Faucitano L, Laforest JP, Rivest J, et al. (2006). Effects of slaughter weight on carcass composition and meat quality in pigs of two different growth rates. Meat Sci. 72: 91-99.

Galián M, Poto A and Peinado B (2009). Carcass and meat quality traits of the Chato Murciano pig slaughtered at different weights. Livest Sci. 124: 314-320.

Honikel KO (1998). Reference methods for the assessment of physical characteristics of meat. Meat Sci. 49: 447-457.

Kim GD, Kim BW, Jeong JY, Hur SJ, et al. (2013). Relationship of carcass weight to muscle fiber characteristics and pork quality of crossbred (Korean native black pig x Landrace) F2 pigs. Food Bioproc. Tech. 6: 522-529.

Li JG, Li JZ and Wang DF (1991). Experiment study on two-way cross and three-way cross between Hanzhong white pig and lean-typy swine. Swine Prod. 4: 29-31.

Park PW and Goins RE (1994). In situ preparation of fatty acid methyl esters for analysis of fatty acid composition in foods. J. Food Sci. 59: 1262-1266.

Raes K, De Smet K and Demeyer D (2004). Effect of dietary fatty acid on incorporation of long chain polyunsaturated fatty acids and conjugated linoleic acid in lamb, beef and pork meat: a review. Anim. Feed. Sci. Technol. 113: 199-221.

Rehfeldt C, Fiedler I, Dietl G and Ender K (2000). Myogenesis and postnatal skeletal muscle cell growth as influenced by selection. Livest. Prod. Sci. 66: 177-188.

Ryu YC and Kim BC (2005). The relationship between muscle fiber characteristics, postmortem metabolic rate, and meat quality of pig longissimus dorsi muscle. Meat Sci. 71: 351-357.

Ryu YC and Kim BC (2006). Comparison of histochemical characteristics in various pork groups categorized by postmortem metabolic rate and pork quality. J. Anim. Sci. 84: 894-901.

Teye GA, Shearda PR, Whittingtona FM, Nutea GR, et al. (2006). Influence of dietary oils and protein level on pork quality. 1. Effects on muscle fatty acid composition, carcass, meat and eating quality. Meat Sci. 73: 157-165.

Zhang Y, Liu LT, Ji C, Ma QG, et al. (2010). The effects of dietary alpha-lipoic acid (LA) level on meat quality and histology characteristics of muscle fiber in broilers. China Feed. 16: 29-33

Zhao JF and Liu J (2007). The breed protection and utilization for Hanjiang River black pig. J. Xinyang Agricult. Coll. 17: 119-121. 